\title{
Investigations of the response of hybrid particle detectors for the Space Environmental Viewing and Analysis Network (SEVAN)
}

\author{
A. Chilingarian and A. Reymers \\ Alikhanyan Physics Institute, Yerevan, Armenia, Alikhanyan Brothers 2, Yerevan 375036, Armenia
}

Received: 29 January 2007 - Revised: 25 September 2007 - Accepted: 25 September 2007 - Published: 26 February 2008

\begin{abstract}
A network of particle detectors located at middle to low latitudes known as SEVAN (Space Environmental Viewing and Analysis Network) is being created in the framework of the International Heliophysical Year (IHY2007). It aims to improve the fundamental research of the particle acceleration in the vicinity of the Sun and space environment conditions. The new type of particle detectors will simultaneously measure the changing fluxes of most species of secondary cosmic rays, thus turning into a powerful integrated device used for exploration of solar modulation effects. Ground-based detectors measure time series of secondary particles born in cascades originating in the atmosphere by nuclear interactions of protons and nuclei accelerated in the galaxy. During violent solar explosions, sometimes additional secondary particles are added to this "background" flux. The studies of the changing time series of secondary particles shed light on the high-energy particle acceleration mechanisms. The time series of intensities of high energy particles can also provide highly cost-effective information on the key characteristics of interplanetary disturbances. The recent results of the detection of the solar extreme events (2003-2005) by the monitors of the Aragats Space-Environmental Center (ASEC) illustrate the wide possibilities provided by new particle detectors measuring neutron, electron and muon fluxes with inherent correlations. We present the results of the simulation studies revealing the characteristics of the SEVAN networks' basic measuring module. We illustrate the possibilities of the hybrid particle detector to measure neutral and charged fluxes of secondary $\mathrm{CR}$, to estimate the efficiency and purity of detection; corresponding median energies of the primary proton flux, the ability to distinguish between neutron and proton initiated GLEs and some other important properties of hybrid particle detectors.
\end{abstract}

Correspondence to: A. Reymers

(arthur@crdlx5.yerphi.am)
Keywords. Interplanetary physics (Cosmic rays; Energetic particles; Instruments and techniques)

\section{Introduction}

High energy particles, accelerated on or near the Sun (Solar Cosmic Rays - SCR), are superimposed on the Galactic Cosmic Ray (GCR) background. These energetic particles pose a serious hazard to humans in space, as well as to the electronics and other important technological resources. Understanding the fundamental physical processes of particle acceleration on the Sun and by coronal and interplanetary (IP) shocks is crucial for the exploration of space.

The size and magnetic field of IP Coronal Mass Ejections (ICMEs) are obviously correlated with the modulation effects that the ICME poses on the galactic cosmic rays during its propagation up to $1 \mathrm{AU}$. On the way to Earth $(15-50 \mathrm{~h})$ a magnetic cloud and shock modulate the GCR flux, changing the flux intensity and making it anisotropic. Reaching the terrestrial atmosphere GCRs initiate particle cascades. Among numerous cascade particles high energy muons approximately follow the primary particle trajectory. The spatial-temporary pattern of the muons on the Earth's surface can be used for "mapping" heliospheric disturbances, i.e. the size and magnetic field of the approaching ICME. Only the highest energy CR (with rigidities $500 \mathrm{MV}-14 \mathrm{GV}$, dependent on the latitude and altitude of the detector location) can penetrate the atmosphere and generate particle cascades reaching the Earth's surface. Because these particles are very fast, the information on the disturbances of the IP magnetic field is rapidly transferred and may prove useful for space weather forecasting.

In the statistical study by Kudela and Storini (2006) the relation of the CR variability/anisotropy with the strength of the geomagnetic storms was investigated. It was demonstrated that the parameters of the changing $\mathrm{CR}$ time series

Published by Copernicus Publications on behalf of the European Geosciences Union. 


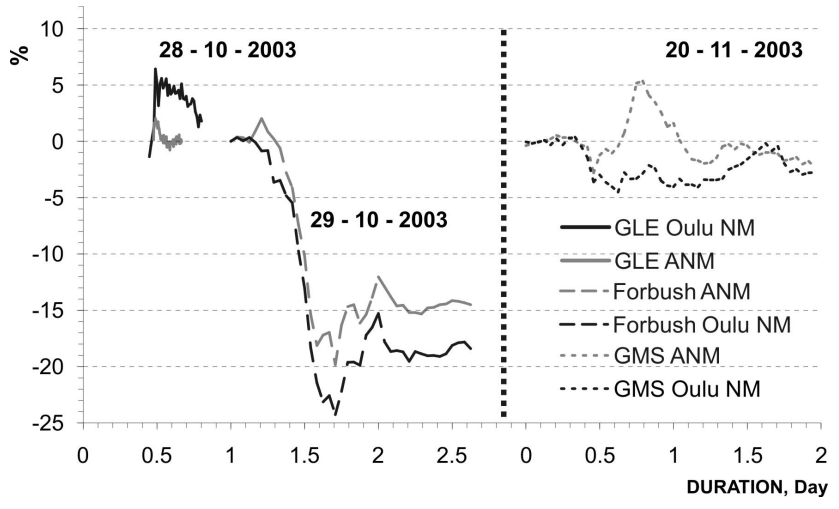

Fig. 1. 2003 Halloween events detected by particle detectors located at high latitude (Oulu NM, vertical cutoff rigidity $\sim 0.8 \mathrm{GV}$, bold black curves) and middle latitude (Aragats NM (vertical cutoff rigidity $\sim 7.1 \mathrm{GV}$, gray curves).

are potentially useful for the geomagnetic activity forecasts. The measurements of the changing fluxes provided by spaceborne spectrometers have excellent energy and charge resolution. Ground-based detectors measure the time series of secondary particles born in cascades originating in the atmosphere by primary ions and solar neutrons. The networks of particle detectors can detect these modulation effects; predict the upcoming geomagnetic storm hours before the ICME arrival at the ACE and SOHO spacecrafts. The less than hour lead time (the time span of ICME travel from spacecraft to magnetosphere), provided the particle detectors located at $\mathrm{ACE}$ and $\mathrm{SOHO}$ at libration point 1.5 million kilometers far from the Earth is too short to take effective mitigation actions and to protect the satellites and surface industries from the harm of major geomagnetic storms.

To establish a reliable and timely forecasting service, we need to measure, simulate and compare:

- the time series of neutrons and high energy muons;

- the correlations between the changing fluxes of various secondary particles; and

- the direction of the detected secondary cosmic rays.

The surface monitors located at the Aragats Space Environmental Center (ASEC) at 2000 and $3200 \mathrm{~m}$ altitudes $\left(40^{\circ} 25^{\prime} \mathrm{N}, 44^{\circ} 15^{\prime} \mathrm{E}\right.$; vertical cut-off rigidity in 2007 : $7.1 \mathrm{GV}$ ) detect charged and neutral components of secondary cosmic rays with different energy thresholds and various angles of incidence (see Table 1; the detailed description of ASEC detectors can be found in Chilingarian et al., 2005).

Using our experience (see Chilingarian et al., 2005, 2003a, b, 2007; Gevorgyan et al., 2005; Chilingarian and Reymers, 2007; Bostanjyan et al., 2007) with data analysis of multivariate time-series from ASEC monitors, we designed and fabricated a new-type of particle detector to meet the above goals. In order to keep the instruments inexpensive, the options are kept flexible by using modular designs. The price of a fully autonomous basic detector, with the facility to send data to the Internet will not exceed \$20000 U.S. For this reason the network of countries involved in space research can be significantly expanded, which will facilitate their part in the International Heliophysical Yyer-2007 (IHY-2007).

At any time one can add additional similar units to achieve improved functionality: for example, one could add several new observational directions and enhance the accuracy of particle fluxes measurements. As a world-wide network of neutron monitors (NM, Moraal et al., 2000), the new monitors will measure neutron fluxes and, additionally, charged particle fluxes with different energy thresholds, thus allowing one to investigate the additional populations of primary ions. These units plan to be deployed at universities and research centers of developing countries to perform survey and monitoring of the most dangerous space storms and to involve new generations of students and scientists in space research.

The network is planned to be installed at middle and low latitudes. It will be compatible with the currently operating high-latitude neutron monitors network "Spaceship Earth" (Kuwabara et al., 2006), coordinated by the Bartol Research Center, with the Solar Neutron Telescopes network coordinated by the Nagoya University (Tsuchiya et al., 2001), with the Muon network coordinated by the group from Shinshu University (Munakata et al., 2000) and with the Athens Neutron Monitor Data Processing Center (Mavromichalaki et al., 2005, 2006).

Figure 1 compares the famous 2003 Halloween events detected by Oulu NM (the vertical cutoff rigidity $\sim 0.8 \mathrm{GV}$, bold black curves) and Aragats NM (vertical cutoff rigidity $\sim 7.1 \mathrm{GV}$, gray curves). The picture shows that the amplitude of the Ground Level Enhancement (GLE) is much bigger at high latitudes ( $\sim 6 \%$ at Ouly and $\sim 2 \%$ on Aragats); Forbush decrease (FD) is also "deeper" at high latitudes; however, the Geomagnetic storm (GMS) is much more pronounced at middle latitudes. The precursors of GSM also start earlier at middle latitudes. Therefore, the SEVAN network will be compatible with the high latitude networks, with enhanced possibilities to alert the satellite operators to extreme radiation and geomagnetic storms.

The potential recipients of particle detectors in this new initiative are Croatia, Slovakia, Costa Rica, Bulgaria, Indonesia, and India (see Fig. 2 and Table 2). When fully deployed the SEVAN network will provide the reliable monitoring of the Sun by a minimum of one detector for $22 \mathrm{~h}$ and by 2 detectors for $18 \mathrm{~h}$ of a day. We assume that particle fluxes measured by the new network at medium to low latitudes, combined with information from satellites and particle detector networks at high latitudes will provide experimental evidence on the most energetic processes in the solar system and will constitute an important element of the global space weather monitoring and forecasting service. In the paper we present the results of the simulation studies, revealing 
Table 1. Characteristics of ASEC monitors.

\begin{tabular}{|c|c|c|c|c|c|}
\hline Detector & $\begin{array}{l}\text { Altitude } \\
\text { m }\end{array}$ & $\begin{array}{c}\text { Surface } \\
\mathrm{m}^{2}\end{array}$ & $\begin{array}{c}\text { Threshold(s) } \\
\text { MeV }\end{array}$ & Operation since & $\begin{array}{c}\text { Count rate } \\
\left(\min ^{-1}\right)\end{array}$ \\
\hline NANM (18NM64) & 2000 & 18 & 50 & 1996 & $2.7 \times 10^{4}$ \\
\hline ANM (18NM64) & 3200 & 18 & 50 & 2000 & $6.1 \times 10^{4}$ \\
\hline ASNT & 3200 & $4(5 \mathrm{~cm}$ thick $)$ & 7 & & $1.2 \times 10^{5}$ \\
\hline 8 channels & 3200 & $4(60 \mathrm{~cm}$ thick $)$ & $85,172,256,382$ & 1998 & $5.2 \times 10^{4 *}$ \\
\hline NAMMM & & 10 - upper & 7 & & $1.8 \times 10^{5}$ \\
\hline 24 channels & 2000 & $10-$ down & 250 & 2006 & $1.1 \times 10^{5}$ \\
\hline AMMM & 3200 & 100 & 5000 & 2002 & $3 \times 10^{5}$ \\
\hline $\begin{array}{l}\text { MAKET-ANI } \\
6 \text { channels }\end{array}$ & 3200 & 6 & 7 & 1996 & $1.5 \times 10^{5}$ \\
\hline
\end{tabular}

*Count rate for the first threshold; near vertical charged particles are excluded

the characteristics of the SEVAN network basic measuring module. We illustrate the possibilities of the hybrid particle detectors to measure neutral and charged fluxes of secondary CR (we estimate the efficiency and purity of detection; determine the corresponding median energies of the primary proton flux), to distinguish between neutron and proton initiated GLEs and some other important properties of hybrid particle detectors.

\section{Calculation of the efficiencies, energy thresholds and count rates of SEVAN particle detectors}

The basic detecting unit of the SEVAN network (see Fig. 3) is assembled from standard slabs of $50 \times 50 \times 5 \mathrm{~cm}^{3}$ plastic scintillators. Between 2 identical assemblies of $100 \times 100 \times 5 \mathrm{~cm}^{3}$ scintillators (four standard slabs) are located two $100 \times 100 \times 5 \mathrm{~cm}^{3}$ lead absorbers and a thick $50 \times 50 \times 25 \mathrm{~cm}^{3}$ scintillator assembly ( 5 standard slabs). A scintillator light capture cone and Photo Multiplier Tube (PMT) are located on the top and bottom, as well as in the intermediate layers of the detector. The detailed detector charts with all sizes are available from http://crdlx5.yerphi.am/.

Incoming neutral particles undergo nuclear reactions in the thick $25-\mathrm{cm}$ plastic scintillator and produce protons and other charged particles. In the upper 5-cm thick scintillator charged particles are registered very effectively; however, for the nuclear interactions of neutral particles there is not enough substance. When a neutral particle traverses the top thin $(5 \mathrm{~cm})$ scintillator, usually no signal is produced. The absence of the signal in the upper scintillators, coinciding with the signal in the middle scintillator, points to neutral particle detection. The coincidence of signals from the top and bottom scintillators indicates the traversal of high energy muons. Lead absorbers improve the efficiency of the neutral flux detection and filtered low energy charged particles. If we denote by " 1 " the signal from the scintillator and by " 0 " the absence of the signal, the following combinations of the 3-layered detector output are possible:

111 - traversal of high energy muon;

011 and 010 - traversal of the neutral particle;

100 - traversal of low energy charged particle stopped in the scintillator or in the first lead absorber.

110 - traversal of higher energy charged particle stopped in the second lead absorber.

001 - registration of the inclined charged particles.

Microcontroller-based Data Acquisition (DAQ) electronics and Advanced Data Analysis System (ADAS) provide registration and storage of all logical combinations of the detector signals for further offline analysis and for online alerts. The slow control system, as on the ADAS subsystem, provides the remote control of the PMT high voltage and the important parameters of the DAQ electronics.

To quantify statements about the detection of different types of particles by the SEVAN modules, we need to perform detailed simulation of the detector response. We use simulated cascades of the charged and neutral secondary particles obtained with the CORSIKA (version 6.204) Monte Carlo code (Heck et al., 1998). The threshold energies for the primary particles assumed as input for CORSIKA correspond to the vertical cutoff rigidity of the detector locations (see Table 2). All secondary particles were tracked until their energy dropped below the predetermined value $(50 \mathrm{MeV}$ for hadrons, $10 \mathrm{MeV}$ for muons and $6 \mathrm{MeV}$ for electrons and photons) or reached all the way to the ground level. The spectra of primary protons and helium nuclei (99\% of the flux at energies up to $100 \mathrm{GeV}$ ) are selected to follow the proton and helium spectra reported by the CAPRICE98 balloon-borne experiment (Boezio et al., 2003).

Among the different species of secondary particles, generated in nuclear-electromagnetic cascades in the atmosphere, muons, electrons, $\gamma$-s, neutrons, protons, pions and kaons were followed by CORSIKA and stored. These particles were used as input for the GEANT3 package (GEANT, 


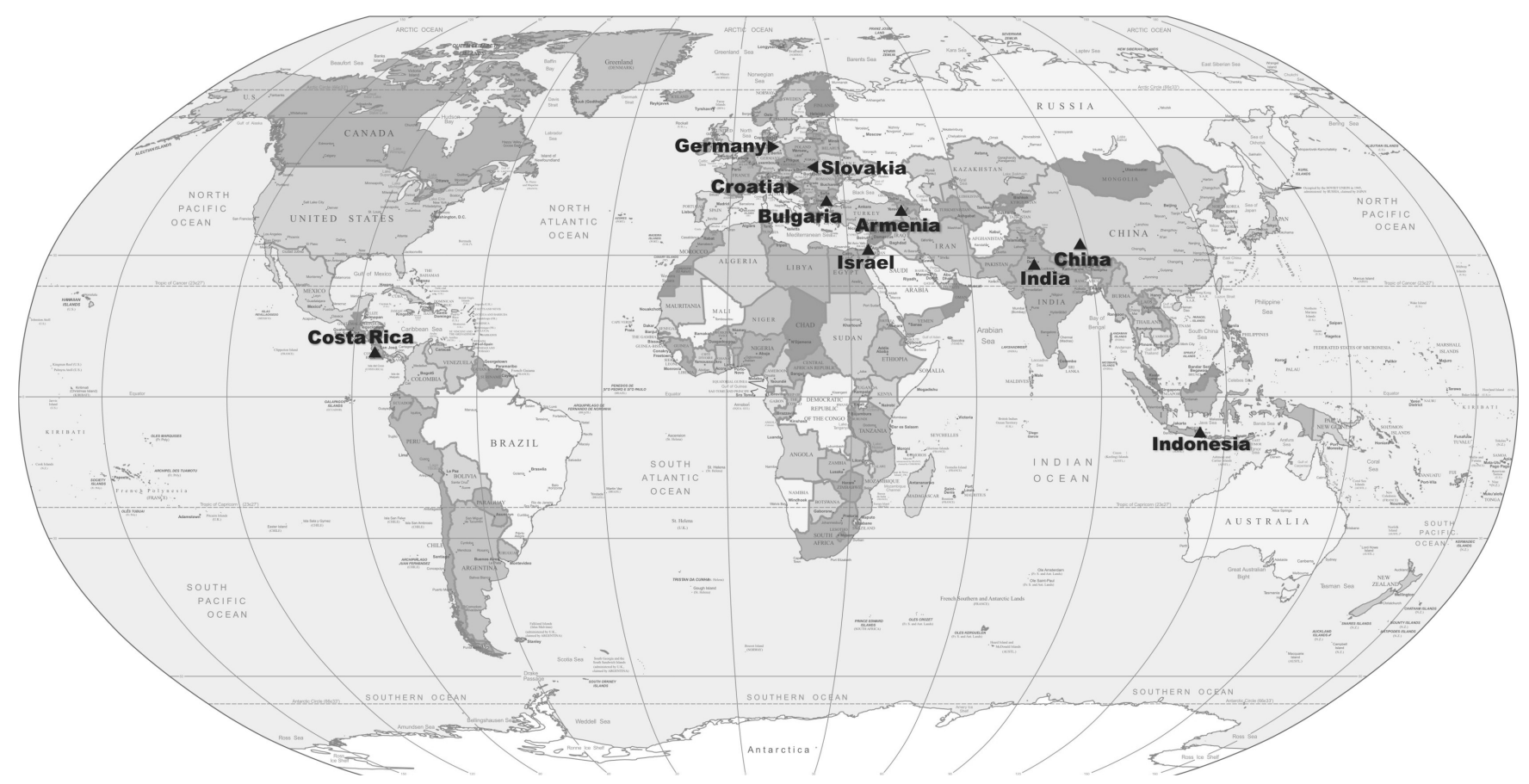

Fig. 2. Possible locations of Space Environment Viewing and Analysis Network (SEVAN).

Table 2. Geophysical characteristics of possible SEVAN sites.

\begin{tabular}{llcccc}
\hline & Station & Latitude & Longitude & Altitude $[\mathrm{m}]$ & $R_{C}(\mathrm{GV})$ \\
\hline Germany & (Greifswald) & $54.5 \mathrm{~N}$ & $13.23 \mathrm{E}$ & 6 & 2.34 \\
Slovakia & (Lomnicky Stit) & $49.2 \mathrm{~N}$ & $20.22 \mathrm{E}$ & 2634 & 3.88 \\
Croatia & (Zagreb) & $45.82 \mathrm{~N}$ & $15.97 \mathrm{E}$ & 120 & 4.89 \\
Bulgaria & (Musala) & $42.1 \mathrm{~N}$ & $23.35 \mathrm{E}$ & 2430 & 6.19 \\
Armenia & (Aragats1) & $40.25 \mathrm{~N}$ & $44.15 \mathrm{E}$ & 3200 & 7.1 \\
Armenia & (Aragats2) & $40.25 \mathrm{~N}$ & $44.15 \mathrm{E}$ & 2000 & 7.1 \\
Israel & (Hermon) & $33.18 \mathrm{~N}$ & $35.47 \mathrm{E}$ & 2025 & 10.39 \\
Costa Rica & (San Jose) & $10.0 \mathrm{~N}$ & $84.0 \mathrm{~W}$ & 1.2 & 10.99 \\
China & (Tibet) & $30.11 \mathrm{~N}$ & $90.53 \mathrm{E}$ & 4300 & 13.86 \\
India & (Delhi) & $28.61 \mathrm{~N}$ & $77.23 \mathrm{E}$ & 239 & 14.14 \\
Indonesia & (Jakarta) & $6.11 \mathrm{~S}$ & $106.45 \mathrm{E}$ & 8 & 16.03 \\
\hline
\end{tabular}

1993), implemented for detector response simulation. Also, we take into account the light absorption in the scintillator (Chilingarian et al., 2007).

The efficiency of the charged particle detection by all 3 layers of the SEVAN detector is about 95\%; the neutron detection efficiency in the middle "thick" scintillator reaches $30 \%$ at $200 \mathrm{MeV}$, the efficiency of the $\gamma$-quanta detection reaches $60 \%$ at the same energies. Responses of all SEVAN detecting layers to the "background" Galactic cosmic rays are presented in Figs. 4-6. The figures show that different layers are sensitive to different particles. While the upper layer is "selecting" mostly electrons and muons, the middle layer is more sensitive to neutrals and the lower layer to the high energy muons. Also, it is apparent from the figures that the best location for the SEVAN detector is at high altitudes, although its location at the sea level would also provide valuable information on neutral and charged fluxes. The lead absorbers filter low energy electrons and gammas. Figure 7 shows that the lower layer is sensitive to the high energy muon flux with the threshold energy $\sim 250 \mathrm{MeV}$.

The middle scintillator of the SEVAN detector intended to select neutrons is also sensitive to $\gamma$-quanta. Figure 5 shows that the fraction of the $\gamma$-quanta in particles detected by the middle thick SEVAN scintillator is higher than the fraction of neutrons for all latitudes. This contamination of the gammas can deteriorate the ability of the SEVAN detector to indicate 


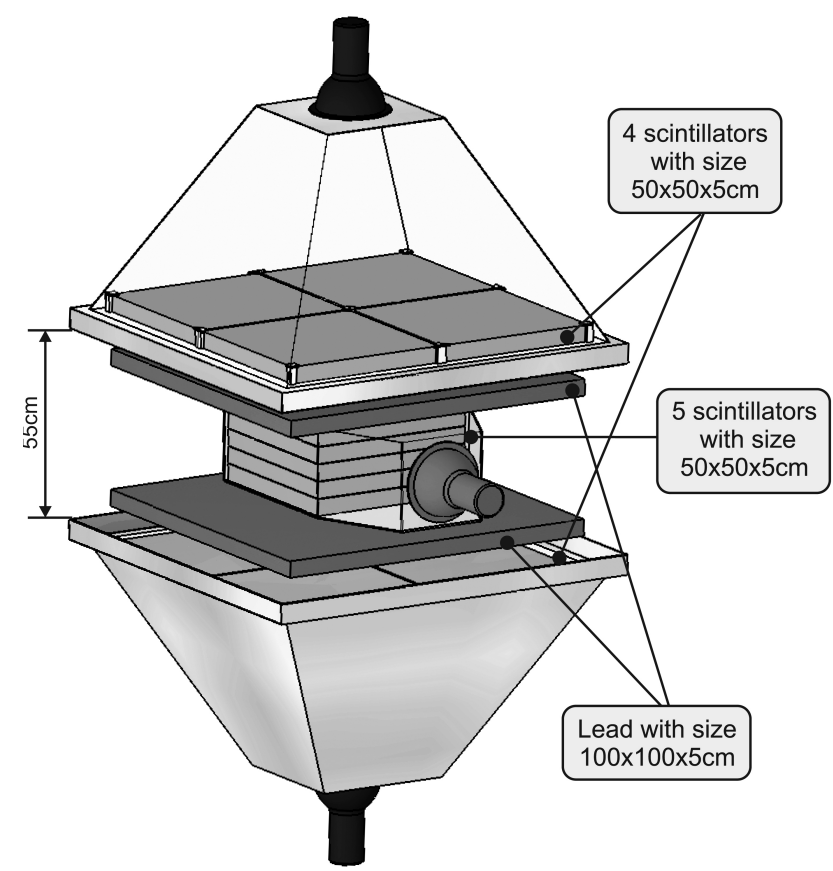

Fig. 3. The basic detecting unit of the SEVAN network.

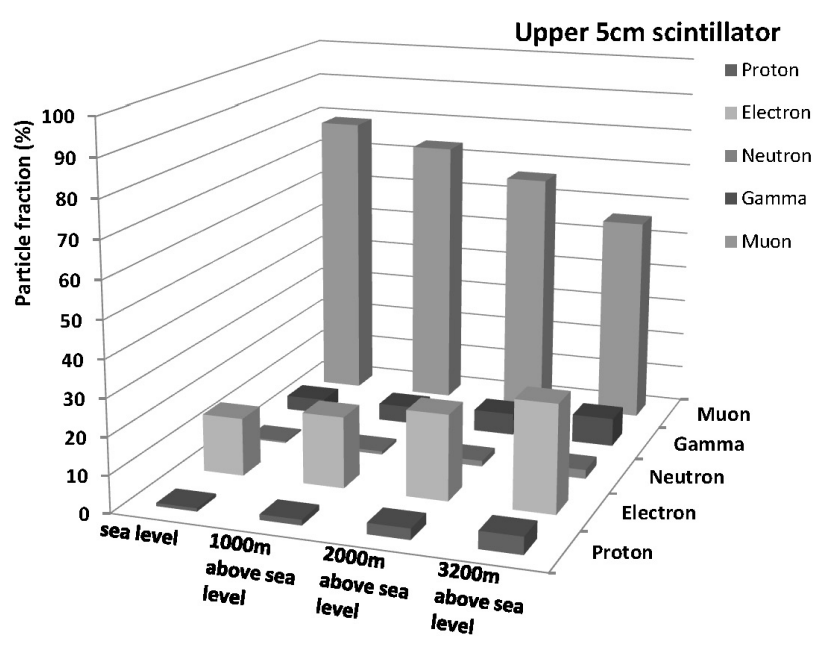

Fig. 4. Fractions of elementary particles detected by the upper layer of the SEVAN detector.

the enhancement of the count rate caused by the additional flux of solar neutrons. The upper 5-cm detector, of course, cannot exclude the neutral gammas, as it is excluded charged muons and electrons. In order to distinguish between the gammas, we test the sandwich system of two 1-cm scintillators with an additional 1-cm layer of lead in between instead of the 5-cm scintillator (see Fig. 8). The 1-cm scintillator under the $1 \mathrm{~cm}$ of lead will effectively detect $\gamma$-quanta by electron-positron pairs generated in lead. In turn, the probability of the nuclear interaction of neutrons in $1 \mathrm{~cm}$ of lead

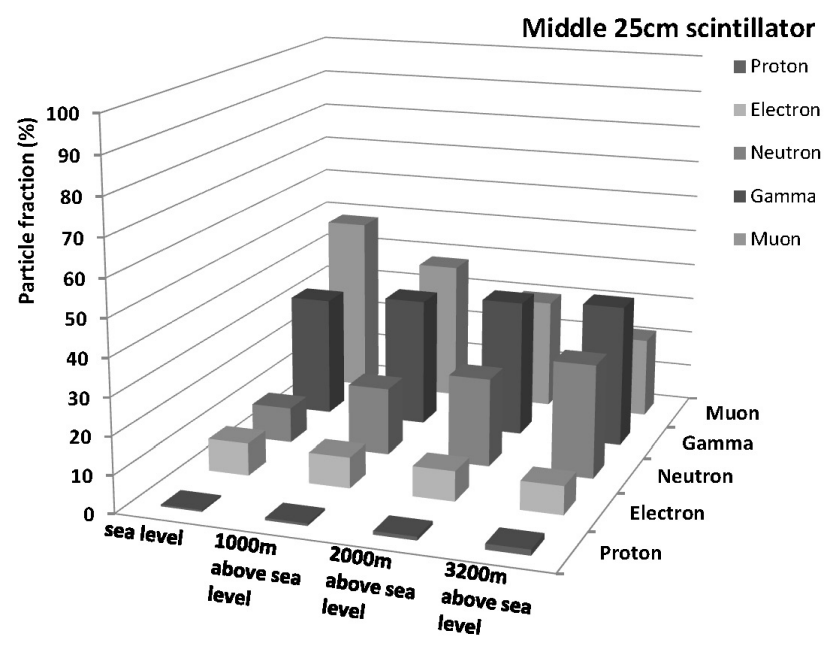

Fig. 5. Fractions of elementary particles detected by the middle layer of the SEVAN detector.

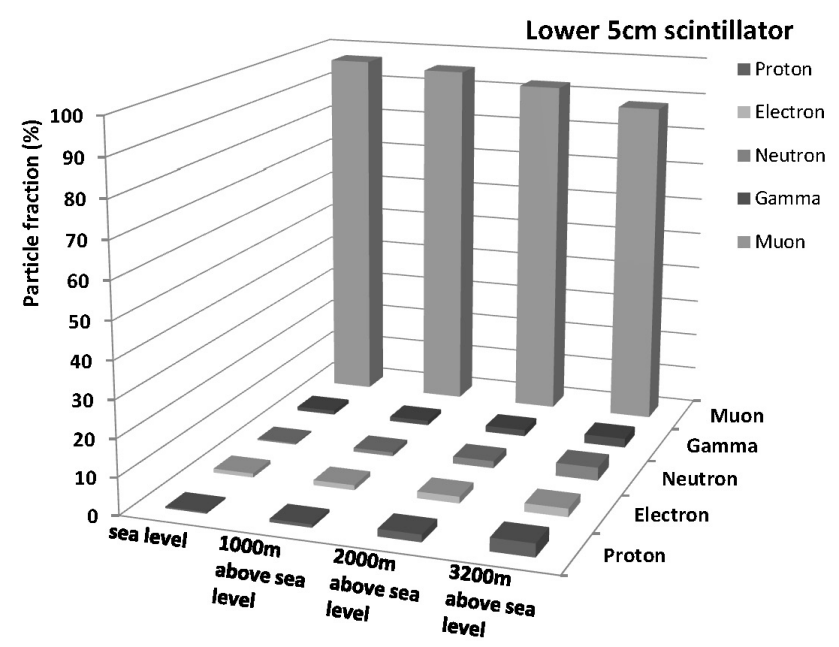

Fig. 6. Fractions of elementary particles detected by the lower layer of the SEVAN detector.

is much less and neutrons will escape detection beneath $1 \mathrm{~cm}$ of lead. Thus, the scintillator above $1 \mathrm{~cm}$ of lead will reject muons and electrons, and the scintillator below $1 \mathrm{~cm}$ of lead will distinguish and reject gammas (see details in Reymers, 2007).

If we again denote by " 1 " the signal from the scintillator and by " 0 " the absence of a signal, the following combinations of the 4-layered detector are of special interest:

1000 - low energy charged particle stopped in $1 \mathrm{~cm}$ of lead;

1100 - low energy charged particle stopped in $5 \mathrm{~cm}$ of lead;

0100 - low energy neutral particle mostly ( $\gamma$-quanta) giving a cascade of $1 \mathrm{~cm}$ of lead absorbed in $5 \mathrm{~cm}$ of lead; 


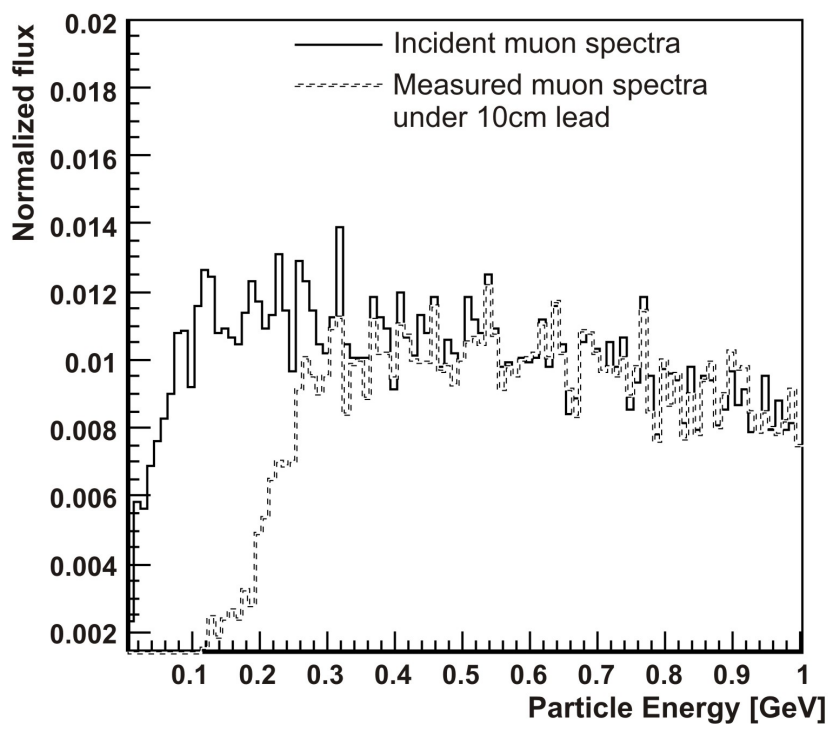

Fig. 7. The energy spectra of muons registered in the upper and bottom 5-cm thick scintillators. The energy threshold of the upper scintillator is $\sim 7 \mathrm{MeV}$, bottom $-\sim 250 \mathrm{MeV}$.

0110 - high energy neutral particle ( $\gamma$-quanta or neutron) giving cascades both in $1 \mathrm{~cm}$ of lead and in $5 \mathrm{~cm}$ of lead;

1110 - charged particle (muon) traversing $6 \mathrm{~cm}$ of lead;

1111 - traversal of high energy muon;

0010 - neutral particle (mostly neutron);

0011 - neutral particle (mostly neutron) generating a large cascade in a thick scintillator.

Table 3 compares the purity of neutron detection by both detector setups. Using the 4-layered detector we not only suppress twice contamination of gamma quanta, but also improve the efficiency of neutron detection. "Resolving" the neutral flux to the neutron and gamma fractions will help to investigate complicated neutron GLE events like the event on 28 November 1998. From Muraki (2005) it is apparent that the knowledge of both gamma and neutron fluxes highly improved the understanding of the event origin.

\section{Calculation of the response of SEVAN particle detec- tors to Galactic and Solar Cosmic Rays (GCR and SCR)}

Fluxes of secondary particles measured by the SEVAN detector are sensitive to different populations of the primary particles incident on the terrestrial atmosphere. Each type of the detected secondary particle (neutron, low energy electrons and muons, high energy muons) is produced by the primary solar and galactic ions having rather broad energy distributions. Nonetheless, the most probable energies corresponding to different secondary particles are shifted relative to each other. In Table 4 the most probable energies of primary protons are presented (medians of the energy distribution of the

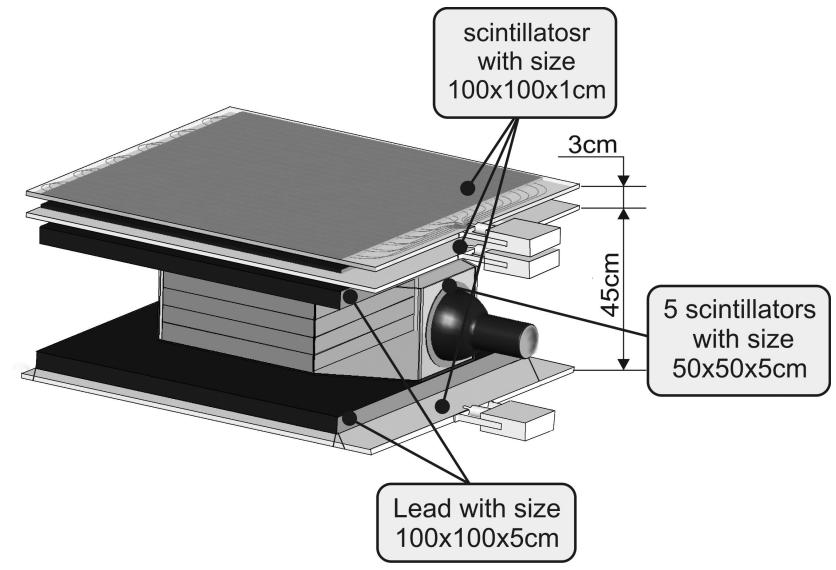

Fig. 8. Four-layer basic unit of the SEVAN.

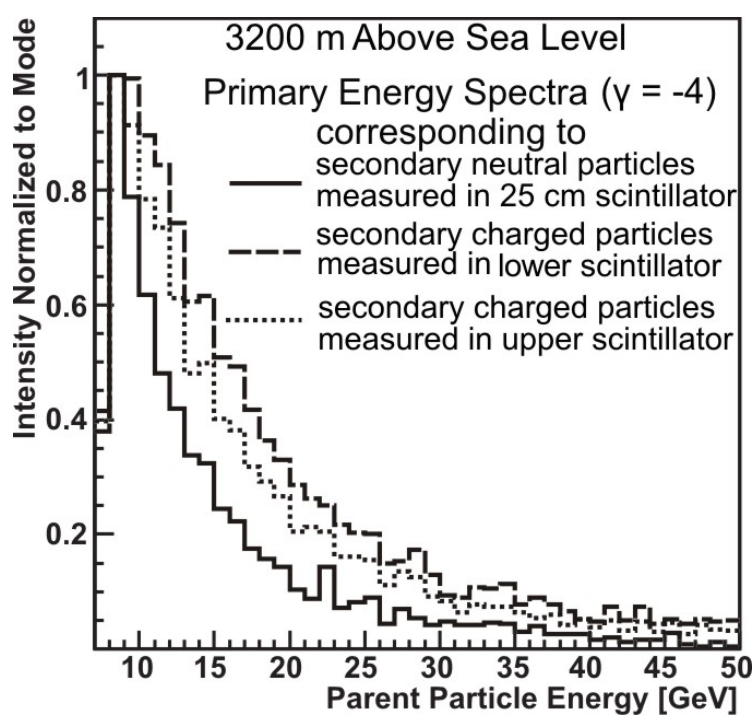

Fig. 9. Spectra of Primary Protons initiated counts in different layers of the SEVAN detector.

parent protons) producing different elementary particles in the terrestrial atmosphere. From the table we can see that secondary muons and electrons are produced by the protons with higher primary energies compared with secondary neutrons and protons.

Table 5 demonstrates the most probable energies of the primary protons "selected" by the layers of the SEVAN detector. The difference of 8.5 and $14.5 \mathrm{GeV}$ of the most probable energy of the primaries is significant if we take into consideration the fact that differential energy spectra of GCRs is rapidly attenuated according to the power law with index of $\sim-2.7$.

In Fig. 9 we present the energy spectra of the primary protons of SCRs selected conditionally on the SEVAN detector layer where the signal was registered. The initial energy spectra were simulated according to the power spectrum $\mathrm{dN} / \mathrm{dE} \sim \mathrm{AE}^{\gamma}$ with spectral index $\gamma=-4$. 
Table 3. Percent of different secondary particles detected by $25-\mathrm{cm}$ thick scintillator in 2 versions of the SEVAN (Aragats station at $3200 \mathrm{~m}$ above sea level).

\begin{tabular}{llcccc}
\hline & Electrons & Muons & Gamma & Neutrons & Protons \\
\hline Veto $5 \mathrm{~cm}$ Scintillator & 6.8 & 16.4 & 40.2 & 35.6 & 1 \\
Veto $1 \mathrm{~cm}$ Scin.+1 cm Lead+1 cm Scin. & 7.4 & 22.3 & 23.7 & 45.3 & 1.3 \\
\hline
\end{tabular}

Table 4. The most probable energies of the Galactic protons corresponding to the different types of secondary particles at $3200 \mathrm{~m}$ above sea level.

\begin{tabular}{lc}
\hline $\begin{array}{l}\text { Secondary particle type } \\
\text { at } 3200 \mathrm{~m}\end{array}$ & $\begin{array}{c}\text { Most probable energy of GCR } \\
(\mathrm{GeV})\end{array}$ \\
\hline Neutron & 8.5 \\
Proton & 8.5 \\
Gamma & 10.5 \\
Electron & 11.5 \\
Muon & 12.5 \\
\hline
\end{tabular}

A large energy spectra index of the SCRs at the highest energies $(\gamma \geq-5$; usually SCR spectra is very steep at $\mathrm{GeV}$ energies: $\gamma \leq-7)$ is a very good indicator of the upcoming abundant SCR protons and ions with energies $>50 \mathrm{MeV}$, extremely dangerous for the astronauts and high transpolar flights, as well as for satellite electronics. Therefore, it is very important to estimate the index of the power law. Lockwood et al. (2002) proposed to estimate the energy spectra index using the data of NM located at the same latitude but at different altitudes. The same methodology has been recently used for the determination of the dose of radiation received on board airplanes during solar particle events (Lantos, 2005) and for estimating the spectral index of the 20 January 2005 GLE (for details of this outstanding event, see, for example, Plainaki et al., 2007) using the data of the Aragats and Nor Amberd NM (Zazyan and Chilingaryan, 2005).

To simulate the proposed method of determination of the spectral index we need to know the spectra of solar neutrons and protons above the atmosphere which are intense enough to generate a significant enhancement at location of the ASEC monitors. The test neutron spectrum at the top of the terrestrial atmosphere was adopted from Watanabe et al. (2006):

$I_{n}(E)=176 E^{-3.6}\left(\mathrm{~m}^{2} \cdot \mathrm{ster} \cdot \mathrm{s} \cdot \mathrm{GeV}\right)^{-1}$,

$E \in(0.1-2 \mathrm{GeV})$

The spectra of secondary particles from the neutron traversal in the terrestrial atmosphere were obtained by the PLANETOCOSMICS code (http://cosray.unibe.ch/ laurent/ planetocosmics/). The majority of the secondary particles at
Table 5. Modes of the GCR energy spectra corresponding to different species of secondary particles registered by the SEVAN detector at $3200 \mathrm{~m}$ above sea level.

\begin{tabular}{lc}
\hline $\begin{array}{l}\text { Layers of detector } \\
\text { located at } 3200 \mathrm{~m}\end{array}$ & $\begin{array}{c}\text { Most probable energy of GCR } \\
(\mathrm{GeV})\end{array}$ \\
\hline Upper Layer & 11.5 \\
Middle 25 cm layer & 8.5 \\
Down Layer & 14.5 \\
\hline
\end{tabular}

$3200 \mathrm{~m}$ are neutrons and gammas. The obtained spectra of the secondary particles coincide well with the ones reported in Shibata (1994).

The test solar proton spectra from $7 \mathrm{GeV}$ (above the cutoff rigidity of the Aragats station) to $30 \mathrm{GeV}$ were adopted from Zazyan and Chilingaryan (2005) as follows:

$$
\begin{aligned}
& I_{p}(E)=4.1 \times 10^{5} E^{-5}\left(\mathrm{~m}^{2} \cdot \mathrm{ster} \cdot \mathrm{s} \cdot \mathrm{GeV}\right)^{-1}, \\
& (E \in 7.1-30 \mathrm{GeV}) .
\end{aligned}
$$

The spectra of secondary particles were obtained in the same way as for the primary neutron flux. The simulated test secondary fluxes were used as the input of GEANT3 code to simulate the detectors response. Proceeding from simulated enhancements initiated by the "proton" and "neutron" GLEs, it is possible to estimate the power law index of the SCR using the ratio of the enhancements of the neutron flux and low energy charged particles. We made simulations of the primary proton flux by changing the power law index from -4 to -6 . Figure 10 shows that the ratio of the neutral-to-charge flux is indeed sensitive to the power index abruptly decreasing with the decrease in the power index.

The comparison of the count rate enhancement in the SEVAN layers allows one to distinguish very rare events with a significant solar neutron flux incident in the terrestrial atmosphere. Table 6 shows that for neutron primaries there is a significant enhancement in the thick layer and much less enhancement in thin layer. For proton primaries the situation is vice-versa: the significant enhancement is in the thin layer, and the thick layer did not demonstrate unambiguous additional flux. 
Table 6. Simulated enhancements (in standard deviations) of the "5-min" count rates corresponding to GLEs initiated by primary neutrons (1) and primary protons (2).

\begin{tabular}{lll}
\hline Detector layer & Solar Protons & Solar Neutrons \\
\hline Upper 5 cm scintillator & $4.8 \sigma$ & $2.6 \sigma$ \\
Middle 25 cm scintillator & $1.7 \sigma$ & $6.4 \sigma$ \\
\hline
\end{tabular}

\section{Conclusion}

The reliable way to forewarn the occurrence of upcoming violent space storms is to scrutinize the solar wind upstream of the Earth. The current source of these data is a NASA research satellite ACE and the ESA-NASA research satellite SOHO, which unfortunately has already outlived its expected period of service. Heliospheric Imager (HI) on board each of the STEREO spacecraft (one leading the Earth, and the other lagging the Earth), provides new opportunities for space weather research. It gives the first chance to observe "geo-effective" ICMEs along the Sun-Earth line in interplanetary space. The consequences of the interaction of the ICME with the magnetosphere can be calculated using HI data in a much more precise way than was possible previously (see details in Harrison et al., 2007). Within NASA's Living With a Star (NASA-LWS) projects, it is planned to launch a constellation of four identically instrumented Inner Heliospheric Sentinels (IHS) to make in-situ measurements of the fluxes of energetic particles as close to the Sun as 0.25 , as well as multipoint remote-sensing observations of solar $\mathrm{X}$-ray, radio, gamma-ray, and neutron emissions (Solar Sentinels project, 2006).

Networks of particle detectors on the Earth's surface will provide complimentary information and will be an important element of planetary space weather warning services. A huge advantage of ground-based particle detectors is their consistency, 24-h coverage, and multi-year operation. In contrast to the planned life of the satellites and spacecraft is of only a few years (STEREO - 2 years), they are affected by the same solar blast for which they should alert, and instead of sending warning the space-born facilities are usually set in the stand-by mode.

Multi-particle detectors proposed in the present paper will probe different populations of primary cosmic rays. The basic detector of the SEVAN network is designed to measure fluxes of neutrons and gammas, of low energy charged component and high energy muons. The rich information obtained from the SEVAN network located mostly at low and middle latitudes, will allow one to estimate the energy spectra of the highest energy SCR. The SEVAN network will be sensitive to very weak fluxes of SCR above $10 \mathrm{GeV}$, a very poorly explored highest energy region. To understand the sensitivity of the new type of particle detectors to the highest

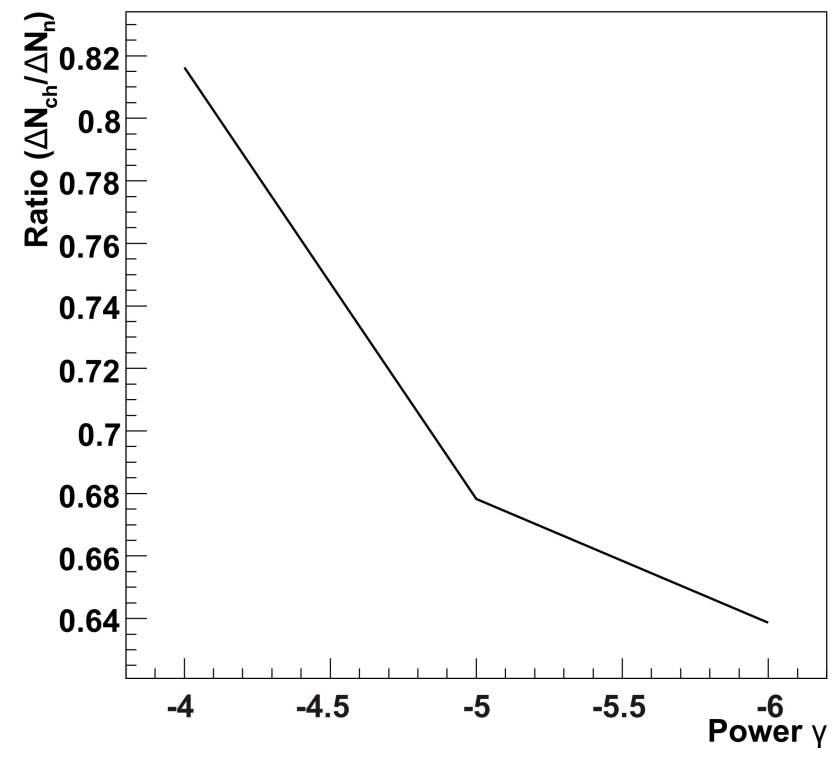

Fig. 10. The ratio of the enhancements of the flux of neutrons and low energy charged particles dependent on the power index of the SCR spectra.

energy solar ions we investigate the response of the SEVAN basic units to galactic and solar protons. The hard spectra of solar ions at highest energies $(\gamma \sim-4$ to -5 at rigidities of $\geq 10 \mathrm{GV}$ ) indicate the upcoming very intense solar ion flux with rigidities $>50 \mathrm{MV}$, very dangerous for satellite electronics and astronauts. The SEVAN network detectors will also allow one to distinguish between very rare and very important GLEs initiated by primary neutrons.

Summarizing, the hybrid particle detectors, measuring neutral and charged fluxes provide following advantages upon existing detector networks measuring single species of secondary CR:

- Enlarge statistical accuracy of measurements;

- Probe different populations of primary CR from $7 \mathrm{GV}$ up to $20-30 \mathrm{GV}$;

- Reconstruct SCR spectra and determine position of the spectral "knees";

- Classify GLEs in "neutron" or "proton" initiated events;

- Estimate and analyze correlation matrices between different fluxes;

Construction of the SEVAN network is started in the framework of the International Heliophysical Year and United Nations Basic Space Science program, focusing on the deployment of arrays of small, inexpensive instruments around the world. The Cosmic Ray Division of the Alikhanyan Physics Institute will donate the scintillators, photomultipliers and 
Data Acquisition electronics to donor countries. Installation of the first SEVAN detector abroad is planned in autumn 2007 in Croatia, supported by the European Office of Aerospace Research and Development (EOARD). Installation of a SEVAN detector in India is planned in 2008, and will be supported by the Asian Office of Aerospace Research and Development (AOARD). Meanwhile several SEVAN detectors are under testing at research high-mountain stations on the slopes of mountain Aragats in Armenia.

Acknowledgements. The authors thank M. Zazyan for providing CORSIKA simulation results. The authors thank H. Martirosyan for preparing Fig. 1. Our work was supported by ISTC A1058 grant and EOARD grant FA 8655-07-01-3014. Authors also thanks D. Rosa, J. Stamenov, K. Kudela, A. Paz, T. Djamaluddin, S. Mukherjee for their interest to be a part of SEVAN network.

Topical Editor R. Forsyth thanks H. Mavromichalaki and two other anonymous referees for their help in evaluating this paper.

\section{References}

Boezio, M., Bonvicini, V., Schiavon, P., et al.: The cosmic-ray proton and helium spectra measured with the CAPRICE98 balloon experiment, Astroparticle Phys., 19, 583-604, 2003.

Bostanjyan, N. Kh., Chilingarian, A. A., Eganov, V. S., et al.: On the production of highest energy solar protons at 20 January 2005, Adv. Space Res., 39, 1456-1459, 2007.

Chilingarian, A. A. and Reymers, A. E.: Particle detectors in solar physics and space weather research, Astropart. Phys., 27, 465472, 2007.

Chilingarian, A., Melkumyan, L., Hovsepyan, G., et al.: The Response Function of the Aragats Solar Neutron Telescope, Nucl. Instr. and Meth. A, 574, 255-263, 2007.

Chilingarian, A., Arakelya, K., Avakyan, K., et al.: Correlated measurements of secondary cosmic ray fluxes by the Aragats Space - Environmental Center monitors, Nucl. Instrum. Methods Phys. Res., Sect. A, 483-496, 2005.

Chilingarian, A., Avakyan, K., Babayan, V., et al.: Aragats SpaceEnvironmental Center: status and SEP forecasting possibilities, J. Phys. G: Nucl. Part. Phys., 29, 939-952, 2003a.

Chilingarian, A., Babayan, V., Bostanjyan, N., et al.: Monitoring and forecasting of the geomagnetic and radiation storms during the 23rd solar cycle: Aragats Regional Space Weather Center, Adv. Space Res., 31, 861-865, 2003b.

GEANT: Detector Description and Simulation Tool, CERN Program Library Long Writeup W5013, CERN, 1993.

Gevorgyan, N., Babayan, V., Chilingarian, A., and Martirosyan, H.: Test alert service against very large SEP events, Adv. Space Res., 36(12), 2351-2356, 2005.

Harrison, R.,Davis, C., Eyles, C., et al.: Searching for Solar Clouds in Interplanetary Space, Space Research Today, 168, 25-31 April 2007.
Heck, D., Knapp, J., Capdevielle, J. N., et al.: CORSICA: A Mote Carlo code to simulate Extensive Air Showers, Forschungszentrum Karlsryhe, FZKA Report 6019, 1998.

Kudela, K. and Storini, M.: Possible tools for space weather issues from cosmic ray continuous records, Adv. Space Res., 37(8), 1443-1449, 2006.

Kuwabara, T., Bieber, J. W., Clem, J., Evenson, P., and Pyle, R.: Development of a ground level enhancement alarm system based upon neutron monitors, Space Weather, 4, S10001, doi:10.1029/2006SW000223, 2006.

Lantos, P.: Radiation dozes potentially received on-board airplane during recent solar particle events, Radiation Protection Dosimetry, 118, 363-374, 2005.

Lockwood, J. A., Debrunner, H., Flukiger, E. O., and Ryan, J. M.: Solar proton rigidity spectra from 1 to $10 \mathrm{GV}$ of selected flare event since 1960, Solar Phys., 208(1), 113-140, 2002.

Mavromichalaki, H., Souvatzoglou, G., Sarlanis, C., et al.: The new Athens center on data processing from the neutron monitor network in real time, Ann. Geophys., 23, 3103-3110, 2005, http://www.ann-geophys.net/23/3103/2005/.

Mavromichalaki, H., Souvatzoglou, G., Sarlanis, C., et al.: Space weather prediction by cosmic rays, Adv. Space Res., 37(6), 1141-114, 2006.

Moraal, H., Belov, A., and Clem, J. M.: Design and co-Ordination of Multi-Station International Neutron Monitor Networks, Space Sci. Rev., 93, 285-303, 2000.

Munakata, K., Bieber, J. W., Yasue, S., et al.: Precursors of geomagnetic storms observed by the muon detector network, J. Geophys. Res. 105, 27 457-27 468, 2000.

Muraki, Y.: Highest Energy Solar Neutrons Detected in the Solar Flare on November 28th 1998, 2nd International Symposium SEE-2005, Nor-Amberd, Armenia, 229-232, 2005.

Plainaki, C., Belov, A., Eroshenko, E., et al.: Modeling ground level enhancements: Event of 20 January 2005, J. Geophys. Res., 112, A04102, doi:10.1029/2006JA011926, 2007.

Reymers, A.: Determination of ground-based detectors response to Solar, Galactic and extragalactic Cosmic Rays, Yerevan Physics Institute after A. Alikhanyan, PhD Thesis, 2007.

Shibata, S.: Propagation of the solar neutron through the atmosphere of the Earth, J. Geophys. Res., 99(A4), 6651-6666, 1994.

Solar Sentinels: Report of the Science and Technology Definition Team, NASA/TM, 2006-214137, 2006.

Tsuchiya, H., Muraki, Y., Masuda, K., et al.: Detection efficiency of a new type of solar neutron detector calibrated by an accelerator neutron beam, NIM, A 463, 183-193, 2001.

Watanabe, K., Gros, M., Stoker, P. H., et al.: Solar neutron events of 2003 October-November, Astrophys. J., 636, 1135-1144, 2006.

United Nations: Comprehensive overview on the worldwide organization of the International Heliophysical Year 2007, Office for Outer Space affairs, UN office at Vienna, p.44, 2006.

Zazyan, M. Z. and Chilingaryan, A. A.: On the possibility to deduce proton energy spectrum of the 20 January 2005 GLE using Aragats and Nor-Amberd neutron monitors data, 2rd International Symposium SEE-2005, Nor-Amberd, Armenia, 200-202, 2005. 